\title{
Medications in Thai Patients with Oral Lichen Planus, Oral Lichenoid Drug Reaction and Glossitis
}

\author{
${ }^{1}$ Napat Nalamliang, ${ }^{2}$ Nichruethai Tangnantachai, ${ }^{3}$ Kobkan Thongprasom
}

\section{ABSTRACT}

Introduction: Medications have been widely used in the dental patients for the treatment of their systemic diseases. In fact, those drugs have some side-effects to many organs and also the oral cavity. The aim of our study was to investigate the relationship between medications and oral lichen planus (OLP), oral lichenoid drug reaction (OLDR) and glossitis (GT) in Thai patients.

Materials and methods: One hundred and thirty-eight cases of Thai patients were included in this study. Medical records of all cases with oral lesions and symptoms referred to the oral medicine clinic during 2007 to 2010 were extracted. Oral lichen planus group consisted of 88 cases, GT 26 cases and OLDR 24 cases. All data were analyzed using SPSS for Windows version 11.5 .

Results: In our study, $75.5 \%$ of patients used more than one medication while single drug used was $24.5 \%$. Antihypertensive drugs were the most commonly used in all groups. Antihypertensive and hypolipidemic drugs were equally taken $22.4 \%$ in OLP patients. Patients with OLDR taking antihypertensive in $54.2 \%$ followed by hypolipidemic (37.5\%), NSAIDs (25\%), hypoglycemic/antiplatelet (16.7\%) each and others $25 \%$. Patients in GT group were also used antihypertensive drugs $35 \%$, NSAIDs $25 \%$, hypolipidemic $20 \%$ respectively.

Conclusion: Most of patients with oral lesions took more than one medication. Antihypertensive drugs were the most commonly used in Thai patients with oral lesions.

Keywords: Antihypertensive, Glossitis, Lichen planus, Lichenoid, Medications, Thai.

How to cite this article: Nalamliang N, Tangnantachai N, Thongprasom K. Medications in Thai Patients with Oral Lichen Planus, Oral Lichenoid Drug Reaction and Glossitis. Int J Experiment Dent Sci 2014;3(2):73-76.

Source of support: Nil

Conflict of interest: None

\footnotetext{
${ }^{1,2}$ Dental Student, ${ }^{3}$ Professor

1,2Department of Oral Medicine, Chulalongkorn University Thailand

${ }^{3}$ Department of Oral Medicine, Faculty of Dentistry, Chulalongkorn University, Thailand
}

Corresponding Author: Kobkan Thongprasom, Professor Department of Oral Medicine, Faculty of Dentistry, Chulalongkorn University, Thailand, Phone: 6622188942, e-mail: kobkan.t@ chula.ac.th

\section{INTRODUCTION}

Nowaday, many of oral lesions have been found to be related with various factors, such as genetics, nutrition, medications, etc. The medications are now increasingly used due to the advance of drug manufactures and patients with systemic diseases. Our study aimed to find the possibilities of relationship between medications used and common oral lesions in Thai patients. We have focused on the oral lesions such as oral lichen planus (OLP), glossitis (GT) and oral lichenoid drug reaction (OLDR).

Oral lichen planus is the most common chronic inflammatory oral mucosal disease of unclear etiology. Some studies believed that this disease related to the cell-mediated immune response. ${ }^{1,2}$ There are many types of OLP; reticular, papular, plaque, atrophic and ulcerative. ${ }^{2}$ Patients always complain of pain, irritation, burning sensation or taste disturbance. However, there is the lichen-like lesion called 'oral lichenoid lesions (OLLs) which is similar to OLP in clinical and histological appearance but OLLs has the specific cause; oral lichenoid contact lesion (OLCL) which cause by the contact of some materials like amalgam or mercury. Oral lichenoid drug reaction which cause by various drugs such as Allopurinol, Carbamazepine, Chloroquine, Dapsone, Ketoconazole, NSAIDs, Propanolol, Tetracycline. ${ }^{3,4}$ The most reliability in diagnosis of OLDR is to see the lesion resolution after withdrawal of the suspected drugs, and the reaction may recur when the patient is rechallenged with the same drug. Oral lichenoid lesion (s) of graft-versushost disease (OLL-GVHD). GVHD is a lesion that arises in recipients of allogeneic hematopoietic stem cell or bone marrow transplantation. Chronic OLL-GVHD usually presents as keratotic white striae or plaques, with areas of erythema, erosion or ulceration. ${ }^{5}$ In this group of our study, only patients diagnosed with OLP and OLDR were included for investigation. Now, there are no medications can effectively treat OLP lesion due to its recalcitrant in nature and the unclear etiology, but the OLDR can be treated by eliminating its cause, such as withdrawal of suspected drugs. One study reported the possible premalignant character of OLP and OLL. ${ }^{6}$ Interestingly, malignant transformations have been reported the occurrence in the OLL group during the mean follow-up of 53.8 months. ${ }^{7}$ Thus, the relationship between medications and lesions in these two lesions are important 
for those patients and it will be useful for management in OLP and OLDR in the future.

Glossitis is the inflammation of the tongue with burning sensation and feeling irritate and sometimes occurs with other abnormalities. Various tongue appearances, such as geographic tongue or benign migratory GT, fissured tongues atrophic GT and foliate papillitis were also presented with burning sensation. The cause of burning sensation is believed that it was the immunologic reaction or low red cell folate levels. ${ }^{8,9}$

\section{MATERIALS AND METHODS}

One hundred and thirty-eight cases with oral lesions and symptoms were referred to the Oral Medicine Clinic at the Faculty of Dentistry, Chulalongkorn University, Bangkok during 2005 to 2010. Medical records of the patients with OLP 88 cases, GT 26 cases, and OLDR 24 cases were analyzed and included in this study. OLP group consisted of 74 females and 14 males. Age ranged from 23 to 86 years (52.5 \pm 1.45 ; mean $\pm \mathrm{SD}$ ) years. GT group consisted of 22 females and 4 males. Age ranged in GT group were from 28 to 89 years $(55.15 \pm 3.09$; mean $\pm \mathrm{SD})$ years. In OLDR group, females were 17, males were 7 cases. Age of the patients in this group ranged from 37 to 74 years $(57.21 \pm 2.20$; mean \pm SD) years. Systemic diseases and medications taking were recorded at the first visit. All patients were diagnosed by clinical and/or histopathological study except GT group was diagnosed by oral findings and clinical symptoms. OLDR were diagnosed by oral lesions that was similar to OLP erupted after using their medications. GT group was diagnosed by pain/burning sensation tongue with/without inflammation. The manifestations of the tongue were diagnosed by oral examinations as the criteria as follows: ${ }^{10,11}$

Fissured tongue: The dorsum of the tongue showed multiple irregular short or long deep central grooves.
Geographic tongue: Red patch lack of papillae and white patches on the dorsum of the tongue while the white areas have either normal or hyperplastic papillae.

Atrophic tongue: The tongue lacks of normal papillae with atrophic area.

Foliate papillitis: The foliate papillae revealed inflammation characterized by swelling or pain.

All data were analyzed using SPSS for Windows version 11.5.

\section{RESULTS}

In OLP group, 58 out of 88 cases (65.91\%) had been taking medications. By history taking, the oral lesions presented in the oral cavities before administration of drugs. Antihypertensive and hypolipidemic were the most common medications used in those OLP patients. Antihypertensive were used in $22.4 \%$, hypolipidemic $22.4 \%$, hypoglycemic $17.2 \%$, NSAIDs $8.6 \%$ others $22.4 \%$. ACE inhibitors were found to be $13.8 \%$ in OLP group, follow by calcium channel blockers, $\beta$-blockers (6.9\%) each, diuretics $3.4 \%$ and others. In GT group, antihypertensive was also the most commonly used in $35 \%$ followed by NSAIDs $25 \%$, hypolipidemic $20 \%$, hypoglycemic and levothyroxine $15 \%$ each, and others $25 \%$. In OLDR group, all cases had been taking various medications. Antihypertensive was also the most medication used in $54.2 \%$, followed by hypolipidemic (37.\%), NSAIDs (25\%), hypoglycemic (15\%), antipletelet (16.7\%) and others (25\%). All data of medications details used in all groups were shown in Table 1. Furthermore, patients in all groups were taking multiple drugs in $75.5 \%$ while single drug used was $24.5 \%$. The data were shown in Table 2 .

\section{DISCUSSION}

In our study, patients with OLP were equally taken antihypertensive and hypolipidemic drugs (13 of 58; 22.4\%).

Table 1: Medications in patients with OLP, GT and OLDR

\begin{tabular}{llll}
\hline Groups $(N)$ & OLP (\%) & GT (\%) & OLDR (\%) \\
\hline No medication (36) & $30(34.09)$ & $6(23.08)$ & - \\
Medications* (102) $^{*}$ & $58(65.91)$ & $20(76.92)$ & $24(100)$ \\
Antihypertensive & $13(22.4)$ & $7(35)$ & $13(54.2)$ \\
ACE inhibitors & $8(13.8)$ & $3(15)$ & $6(25)$ \\
$\beta$-blockers & $4(6.9)$ & $2(10)$ & $3(12.5)$ \\
Calcium channel blockers & $4(6.9)$ & $5(25)$ & $5(20.8)$ \\
Diuretics & $2(3.4)$ & $2(10)$ & $3(12.5)$ \\
Others & $2(3.4)$ & $1(5)$ & - \\
Hypolipidemic & $13(22.4)$ & $4(20)$ & $9(37.5)$ \\
Hypoglycemic & $10(17.2)$ & $3(15)$ & $44(16.7)$ \\
NSAIDs & $5(8.6)$ & $5(25)$ & $6(25)$ \\
Platelet inhibitors & - & - & $4(16.7)$ \\
Others & $13(22.4)$ & $5(25)$ & $6(25)$ \\
\hline
\end{tabular}

*One patient taking more than one medication, ${ }^{\circledR}$ unspecified medication 
Table 2: Medications using in patients with OLP, OLDR and GT

\begin{tabular}{lllll}
\hline Groups $(N)$ & No medication & Medication (\%) & Single (\%) & Multiple (\%) \\
\hline OLP (88) & $30(21.7)$ & $58(43.0)$ & $17(29.3)$ & $41(70.7)$ \\
OLDR $(24)$ & - & $24(17.4)$ & $3(12.5)$ & $21(87.5)$ \\
GT $(26)$ & $6(4.4)$ & $20(14.5)$ & $5(25)$ & $15(75)$ \\
\hline Total $(138)$ & $36(26.1)$ & $102(73.9)$ & $25(24.5)$ & $77(75.5)$ \\
\hline
\end{tabular}

Similarly, ACE inhibitors were also the most medication using in 8 of 13 cases. One study showed that the prevalence of dyslipidemia was significantly higher in patients with LP. ${ }^{12}$ Moreover, the previous report confirmed that the patients OLP sometimes induced by drugs. ${ }^{13}$ Our findings confirmed that the patients taking antihypertensive and hypolipidemic drugs have been found to be associate with OLP. In fact, the oral lesions in OLP group erupted before taking those medications, so this was the reason why those patients were included in OLP group not in OLDR group.

Regarding OLDR group, all oral lesions erupted after taking medications. Moreover, one patient had taken more than one medication, sometimes it was difficult to know the certain drugs that cause OLDR. Of 54.2\% OLDR patients had been taking antihypertensive drugs and $37.5 \%$ taking hypolipidemic drugs. Six of $24(25 \%)$ patients had been taking NSAIDs that showed OLDR can be caused by the antihypertensive, hypolipidemic and NSAIDs. Medications taking in OLDR patients in our study was similar to the list of drugs in previous report particularly ACE inhibitors, $\beta$-blockers, NSAIDs, etc. ${ }^{3,4,14}$ Interestingly, ACE inhibitors were commonly used in 6 out of 13 cases, following by calcium channel blockers, $\beta$-blockers and diuretics respectively. One study showed that cardiovascular drugs associated in OLL patients $3.6 \%$ that the percentage was lower than in our patients in our study. ${ }^{15}$ However, there is no specific test to confirm diagnosis of OLDR, the resolution of oral lesion after withdrawal of the putative drug and oral lesion eruption when the same drug is administration are dangerous for the patients. Hence, diagnosis of OLDR will base on clinical manifestation, history of medication taking and histopathological features. ${ }^{16}$

Moreover in GT patients, antihypertensive were used in 7 of 20 (35\%), NSAIDs (5 of 20;25\%), hypolipidemic (4 of $20 ; 20 \%$ ) followed by hypoglycemic, levothyroxine and others and respectively. However, antihypertensive drugs are widely used in Asia and Thailand due to the high rate of hypertensive status awareness (56.6\%) and $85.8 \%$ of them were on the treatment. ${ }^{17}$ This study also confirmed that patients with oral lesions used multiple drugs more than single drug and showed the possible relationship between antihypertensive and oral lesions. However, it seems to be difficult to definitely conclude the relationship between the oral lesions and medications. We categorized the unclarify name of drugs or the name of drug was not recorded in the medical charts to the 'others'. Hence, the data we collected in our study was only the definite data. Large group study is needed to clarify this issue. Mouth acts as a window to lot of systemic diseases and serves as a port of entry of the various infections that can alter and affect the immune status of the person. ${ }^{18}$

\section{ACKNOWLEDGMENTS}

This research was support by the Dental Student of Faculty of Dentistry, Chulalongkorn University Grant year 2013. We would like to thank Dr Kevin Tompkin for editing this manuscript. We also thank the Oral Medicine Department staff for their assistant.

\section{REFERENCES}

1. Thornhill MH. Immune mechanisms in oral lichen planus. Acta Odont Scand 2001;59(3):174-177.

2. Lodi G, Scully C, Carozzo M, Griffiths M, Sugerman PB, Thongprasom K. Current controversies in oral lichen planus: Report on an international consensus meeting. Part 1. Viral infections and etiopathogenesis. Oral Surg Oral Med Oral Pathol Oral Radiol Endod 2005;100(1):40-51.

3. Al-Hashimi I, Schifter M, Lockhart P, et al. Oral lichen planus and oral lichenoid lesions: diagnostic and therapeutic considerations. Oral Surg Oral Med Oral Pathol Oral Radiol Endod 2007;103 (Suppl):S25.e1-S25.e12.

4. Do Prado RF, Marocchio LS, Felipini RC. Oral lichen planus versus oral lichenoid reaction: difficulties in the diagnosis. Indian J Dent Res 2009;20(3):361-364.

5. Woo SB, Lee SJ, Schubert MM. Graft-vs-host-disease. Crit Rev Oral Biol Med 1997;8(2):201-216.

6. van der Meij EH, Schepman KP, van der Waal I. The possible premalignant character of oral lichen planus and oral lichenoid lesions: a prospective study. Oral Surg Oral Med Oral Pathol Oral Radiol Endod 2003;96(2):164-171.

7. van der Meij EH, Mast H, van der Waal I. The possible premalignant character of oral lichen planus and oral lichenoid lesions: a prospective five-year follow-up study of 192 patients. Oral Oncol 2007;43(8):742-748.

8. Dawson TAJ. Microscopic appearance of geographic tongue. Br J Dermatol 1969;81(11):827.

9. Taebunpakul P, Piboonratanakit P, Thongprasom K. Folate levels in burning tongue. Acta Stomatol Croat 2007;41(3): 233-240.

10. Rogers RS, Bruce AJ. The tongue in clinical diagnosis. J Eur Acad Dermatol Venereol 2004;18(3):254-259. 
11. Byrd JA, Bruce AJ, Rogers RS. Glossitis and other tongue disorders. Dermatol Clin 2003;21(1):123-134.

12. Dreiher J, Shapiro J, Cohen AD. Lichen planus and dyslipidaemia: a case-control study. Br J Dermatol 2009;161(3):626-629.

13. Scully C, Beyli M, Ferreiro MC, et al. Update on oral lichen planus: etiopathogenesis and management. Criv Rev Oral Biol Med 1998;9(1):86-122.

14. Thompson DF, Skaehill PA. Drug-induced lichen planus. Pharmacotherapy 1994;14(5):561-571.
15. Habbab KM, Moles DR, Porter SR. Potential oral manifestations of cardiovascular drugs. Oral Dis 2010;16(8):769-773.

16. Khudhur AS, Di Zenzo G, Carrozzo M. Oral lichenoid tissue reactions: diagnosis and classification. Expert Rev Mol Diagn 2014;14(2):169-184.

17. Krishman A, Garg R, Kahandailyanage A. Hypertension in the South-East Asia Region. Regional Health Forum 2013;17(10):7-14.

18. Saini R, Saini S, Sharma S. Oral sex, oral health and orogenital infections. J Global Infect Dis 2010;1:57-62. 